\title{
Prospects in Classical Nova Modeling and Nucleosynthesis
}

\author{
Jordi Joséa
}

${ }^{a}$ Departament de Física i Enginyeria Nuclear, Universitat Politècnica de Catalunya, and Institut d'Estudis Espacials de Catalunya (IEEC-UPC), Barcelona, Spain

Classical novae are fascinating stellar events, at the crossroads of astrophysics, nuclear physics and cosmochemistry. In this review, we outline the history of nova modeling with special emphasis on recent advances and perspectives in multidimensional simulations. Among the topics that are covered, we analyze the interplay between nova outbursts and the Galactic chemical abundances, the synthesis of radioactive nuclei of interest for gamma-ray astronomy, such as ${ }^{7} \mathrm{Li},{ }^{22} \mathrm{Na}$ or ${ }^{26} \mathrm{Al}$, and the recent discovery of presolar meteoritic grains, likely condensed in nova shells.

\section{Nuclear ashes: Classical novae and Galactic nucleosynthesis}

Classical novae are close binary systems consisting of a white dwarf, and a large main sequence (or a more evolved) star. The companion overfills its Roche lobe and matter flows through the inner Lagrangian point, leading to the formation of an accretion disk around the compact star. A fraction of this (H-rich) matter ultimately ends up on top of the white dwarf, where it is gradually compressed up to the point when ignition conditions to drive a thermonuclear runaway (hereafter, TNR) are reached.

The thermonuclear origin of nova outbursts was first theorized by Schatzman 36 37. Modern multiwavelength observations and numerical simulations (pioneered by the early hydro models of Starrfield et al. [40]) have drawn a basic picture, usually referred to as the thermonuclear runaway model. Since then, several groups have attempted to improve our understanding of these dramatic stellar events, including state-of-the-art nova nucleosynthesis studies with spherically symmetric (or 1-D) hydro codes (see [262143], and references therein) or preliminary multi-D approaches [1314 24 25].

Nuclear physics plays a crucial role in the course of the explosion. As material from the accretion disk piles up on top of the (CO or ONe) white dwarf, the first nuclear reactions take place. This follows a rise in temperature since degenerate conditions unable the star to readjust the hydrostatic equilibrium by an envelope expansion and, as a result, a TNR ensues. The triggering reaction is ${ }^{12} \mathrm{C}(\mathrm{p}, \gamma)$, which initiates the 'cold' $\mathrm{CNO}$ cycle. At very early stages of the explosion, the main nuclear activity is driven by ${ }^{12} \mathrm{C}(\mathrm{p}, \gamma){ }^{13} \mathrm{~N}\left(\beta^{+}\right){ }^{13} \mathrm{C}(\mathrm{p}$, $\gamma{ }^{14} \mathrm{~N}$. But as the temperature rises, the characteristic time for proton capture reactions on ${ }^{13} \mathrm{~N}$ becomes shorther than its $\beta^{+}$decay time, initiating the 'hot' CNO cycle. This is accompanyied by proton capture reactions onto ${ }^{14} \mathrm{~N}$, leading to ${ }^{15} \mathrm{O}$, as well as by ${ }^{16} \mathrm{O}(\mathrm{p}, \gamma){ }^{17} \mathrm{~F}$ (near peak temperature, $\left.T_{\text {peak }}\right)$. At this stage, the envelope exhibits the presence of significant amounts of ${ }^{13} \mathrm{~N},{ }^{14,15} \mathrm{O}$ and ${ }^{17} \mathrm{~F}$. Indeed, it is the decay of these 
short-lived, $\beta^{+}$unstable nuclei, carried away from the hot envelope's base to the outer, cooler layers by convective transport, what powers the ejection phase [40: their sudden release of energy, few minutes after the peak of the explosion, increases the entropy and temperature of the material. As a result, the electron degeneracy is lifted and an overall expansion sets in, driving the ejection of most (if not all) of the accreted envelope into the interstellar medium (indeed, the TNR is halted by the expansion of the envelope rather than by fuel consumption). The ejected layers enclose the history of multiple nuclear processes that modified its chemical composition during the course of the TNR: they are characterized by huge amounts of the daughther nuclei ${ }^{15} \mathrm{~N},{ }^{17} \mathrm{O}$, and to some extent, ${ }^{13} \mathrm{C}$, which have been claimed to represent the fingerprints of classical nova outbursts in the overall Galactic history (262143, and references therein). Moreover, the envelope is also enriched in other species (depending on the nova type, the mass-accretion rate or the white dwarf initial luminosity), that may contribute to the Galactic abundances to a lesser extent. This includes nuclei such as ${ }^{7} \mathrm{Li},{ }^{19} \mathrm{~F}$, or ${ }^{26} \mathrm{Al}$.

In order to match the energetics, peak luminosities, and associated nucleosynthesis of the so-called fast novae, the modeling of the explosion requires mixing between the solarlike material transferred from the companion and the outermost layers (CO- or ONe-rich) of the underlying white dwarf. In fact, at the typical temperatures expected during the course of the TNR, the amount of leakage from the CNO cycle is very limited [21, and hence, the observed abundances of elements ranging from $\mathrm{Ne}$ to $\mathrm{Ca}$ (significantly overproduced with respect to solar proportions in some novae) cannot be explained in a natural way in terms of nuclear processes. Indeed, the quest for a self-consistent mixing mechanism has become the Holy Grail of nova modeling: several mechanisms have been proposed so far, including diffusion induced convection [30 19], shear mixing [29 28], convective overshoot induced flame propagation [44, convection induced shear mixing [27, or more recently, mixing by gravity wave breaking on white dwarf surfaces 331 . But the final word has not yet been said...

Among the species synthesized during classical nova outbursts, several radioactive nuclei have deserved special attention, in particular those associated with the predicted gammaray output from novae. Whereas ${ }^{13} \mathrm{~N}$ and ${ }^{18} \mathrm{~F}$ may be responsible for a prompt $\gamma$-ray emission at and below $511 \mathrm{keV},{ }^{7} \mathrm{Be}\left({ }^{7} \mathrm{Li}\right)$ and ${ }^{22} \mathrm{Na}$ [1517, which decay much later, may power line emission at 478 and $1275 \mathrm{keV}$, respectively. ${ }^{26} \mathrm{Al}$ is another interesting radioactive isotope that can be synthesized during nova outbursts, although due to its long lifetime only its cumulative emission can be observed. A detailed account of the predicted gamma-ray signatures of classical nova outbursts (including detectability distances) can be found in [18. Hereafter, we will concentrate on the most relevant aspects of nova modeling, pointing out where do we stand and what is missing in our understanding of the overall phenomenon. In particular, we will focus on the synthesis paths of ${ }^{7} \mathrm{Li},{ }^{22} \mathrm{Na}$ and ${ }^{26} \mathrm{Al}$, the associated nucleosynthesis and observational constraints.

\section{1. ${ }^{7} \mathrm{Li}$}

Recent hydrodynamic simulations [1621] have confirmed the feasibility of the $B e$ transport mechanism [5] as the key for ${ }^{7} \mathrm{Li}$ production in nova outbursts: the process is initiated by the synthesis of ${ }^{7} \mathrm{Be}$ through ${ }^{3} \mathrm{He}(\alpha, \gamma){ }^{7} \mathrm{Be}$, which is ultimately transformed into ${ }^{7} \mathrm{Li}(\tau \sim 77$ days $)$ by means of an electron capture, with the emission of a charac- 
teristic $478 \mathrm{keV} \gamma$-ray photon. Huge ${ }^{7} \mathrm{Li}$ overproduction factors (i.e., $\sim 900$ ) with respect to solar values have been obtained, in particular for novae hosting $\mathrm{CO}$ white dwarf cores. These studies stressed the critical role played by the quasi-equilibrium between ${ }^{7} \mathrm{Be}$ and ${ }^{8} \mathrm{~B}$, driven by efficient photodisintegration reactions on ${ }^{8} \mathrm{~B}$, on the survival of ${ }^{7} \mathrm{Be}$ around peak temperatures, and confirmed novae as likely ${ }^{7} \mathrm{Li}$ factories [4] (earlier hydrodynamic simulations assumed, however, envelopes in-place, thus neglecting the possible impact of the initial stages of the TNR and the onset of convective transport on ${ }^{7} \mathrm{Li}$ production). Moreover, they refuted the conclusions based on simple 1- and/or 2-zone models 4 that stressed the key role played by ${ }^{8} \mathrm{~B}(\mathrm{p}, \gamma)$ in breaking the quasi-equilibrium between ${ }^{7} \mathrm{Be}$ and ${ }^{8} \mathrm{~B}$ (and hence, leading to ${ }^{7} \mathrm{Be}$ destruction instead). No nuclear uncertainties in the domain of nova temperatures significantly affect ${ }^{7} \mathrm{Li}$ synthesis [16].

It is worth noting that the potential contribution of classical novae to the Galactic ${ }^{7} \mathrm{Li}$ content turns out to be small (i.e., less than 15\%, according to [1621]). However, a nova contribution seems to be required to match the ${ }^{7} \mathrm{Li}$ content in realistic calculations of Galactic chemical evolution [32].

\section{2. ${ }^{22} \mathrm{Na}$}

The role of ${ }^{22} \mathrm{Na}$ for diagnosis of nova outbursts was first outlined in the seminal work of Clayton \& Hoyle 6. This isotope decays into a short-lived excited state of ${ }^{22} \mathrm{Ne}$, which de-excites to its ground state by emitting a $\gamma$-ray photon of $1.275 \mathrm{MeV}$.

The synthesis of ${ }^{22} \mathrm{Na}$ in novae proceeds through two alternative reaction paths. In the Ne-enriched envelopes of ONe novae [22], it takes place through ${ }^{20} \mathrm{Ne}(\mathrm{p}, \gamma){ }^{21} \mathrm{Na}$, followed either by another proton capture and then, a $\beta^{+}$-decay into ${ }^{22} \mathrm{Na},{ }^{21} \mathrm{Na}(\mathrm{p}, \gamma)^{22} \mathrm{Mg}\left(\beta^{+}\right)^{22} \mathrm{Na}$, or decaying first into ${ }^{21} \mathrm{Ne}$ before another proton capture ensues, ${ }^{21} \mathrm{Na}\left(\beta^{+}\right)^{21} \mathrm{Ne}(\mathrm{p}, \gamma){ }^{22} \mathrm{Na}$. The main destruction channel at nova temperatures is ${ }^{22} \mathrm{Na}(\mathrm{p}, \gamma)^{23} \mathrm{Mg}$.

The nuclear uncertainties associated with the synthesis of ${ }^{22} \mathrm{Na}$ in novae [22] have been recently reduced due to the first direct measurement of the ${ }^{21} \mathrm{Na}(\mathrm{p}, \gamma)$ rate with the DRAGON recoil separator at TRIUMF [38, and to indirect determinations of the ${ }^{22} \mathrm{Na}(\mathrm{p}, \gamma)$ rate carried out with the Gammasphere at the Argonne National Lab [20].

\section{3. ${ }^{26} \mathrm{Al}$}

${ }^{26} \mathrm{Al}$ was discovered in the interstellar medium by the HEAO-3 satellite, through the detection of the $1809 \mathrm{keV} \gamma$-ray line. This characteristic $\gamma$-ray feature is produced by the $\beta^{+}$decay $(\tau=1.04 \mathrm{Myr})$ of the ${ }^{26} \mathrm{Al}$ ground state to the first excited state of ${ }^{26} \mathrm{Mg}$, which in turn de-excites to its ground state level by emitting a $1809 \mathrm{keV}$ photon.

The synthesis of ${ }^{26} \mathrm{Al}$ requires moderate peak temperatures, of the order of $T_{\text {peak }} \leq 2 \times$ $10^{8} \mathrm{~K}$, and a fast decline from maximum temperatures, conditions that are achieved in typical nova outbursts 314322 . ${ }^{26} \mathrm{Al}$ synthesis proceeds through ${ }^{24} \mathrm{Mg}(\mathrm{p}, \gamma){ }^{25} \mathrm{Al}\left(\beta^{+}\right){ }^{25} \mathrm{Mg}(\mathrm{p}$, $\gamma)^{26} \mathrm{Al}^{g}$, whereas it is mainly destroyed by $(\mathrm{p}, \gamma)$ reactions 22 . A significant nuclear uncertainty affects the ${ }^{25} \mathrm{Al}(\mathrm{p}, \gamma){ }^{26}$ Si rate [722], which translates into an uncertainty in the expected contribution of novae to the Galactic ${ }^{26} \mathrm{Al}$ content. Calculations based on recent prescriptions for the composition of ONe white dwarf cores suggest that the contribution of classical nova outbursts to the Galactic ${ }^{26} \mathrm{Al}$ abundance is small (i.e., less than 15\%), in agreement with the results derived from the COMPTEL/CGRO map of the $1809 \mathrm{keV}$ ${ }^{26} \mathrm{Al}$ emission in the Galaxy (see [10]), which points towards young progenitors. 


\section{Observational constraints: from nova shells to presolar grains}

The theoretical nucleosynthetic predictions described above can be compared with the abundance patterns inferred from observations of ejected nova shells (see 2143, and references therein). The comparison yields in general good agreement between models and observations: this includes atomic abundance determinations - $\mathrm{H}, \mathrm{He}, \mathrm{C}, \mathrm{O}, \mathrm{Ne}, \mathrm{Na}$...-, as well as a plausible endpoint for nova nucleosynthesis (around $\mathrm{Ca}$ ), suggesting that the thermal history of the explosion (i.e., $T_{\text {peak }}$, exposure times...) is reasonably well reproduced by current models. Unfortunately, observations provide only direct information on atomic abundances and hence, do not pose severe constraints on the models.

Indeed, better constraints can be (partially) obtained from the laboratory analysis of presolar grains, which yields isotopic abundance ratios. Classical novae are stellar dust factories: infrared measurements in a number of recent novae reveal the presence of Crich dust (Novae Aql 1995, V838 Her 1991, PW Vul 1984...), SiC (Novae Aql 1982, V842

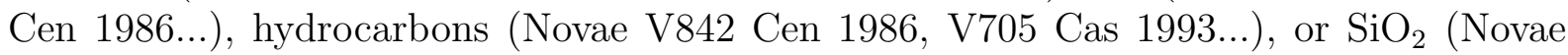
V1370 Aql 1982, V705 Cas 1993). Remarkable examples, such as novae QV Vul 1987, exhibit simultaneous formation of all those types of dust (see [12] for details on dustforming novae). Recently, several characteristic nova signatures have been identified by laboratory isotopic measurements in five silicon carbide and two graphite grains isolated from the Murchison and Acfer 094 meteorites 22. These tiny spherules, only a few microns in size, are characterized by low ${ }^{12} \mathrm{C} /{ }^{13} \mathrm{C}$ and ${ }^{14} \mathrm{~N} /{ }^{15} \mathrm{~N}$ ratios, ${ }^{30} \mathrm{Si}$ excesses and close-to- or slightly lower-than-solar ${ }^{29} \mathrm{Si} /{ }^{28} \mathrm{Si}$ ratios. In some cases, high ${ }^{26} \mathrm{Al} /{ }^{27} \mathrm{Al}$ and low ${ }^{20} \mathrm{Ne} /{ }^{22} \mathrm{Ne}$ ratios have been also determined. This discovery represents the first direct evidence of grains that exhibit nova signatures and opens up interesting possibilities for the future.

Theoretical efforts to predict the expected imprints of nova outbursts on presolar grains have been conducted by different authors [4223], including preliminary estimates on equilibrium condensation sequences in the ejected shells 23]: these studies suggest that classical novae may contribute to the known presolar corundum $\left(\mathrm{Al}_{2} \mathrm{O}_{3}\right)$, spinel $\left(\mathrm{MgAl}_{2} \mathrm{O}_{4}\right)$, enstatite $\left(\mathrm{MgSiO}_{3}\right)$, silicon carbide $(\mathrm{SiC})$ and silicon nitride $\left(\mathrm{Si}_{3} \mathrm{~N}_{4}\right)$ grain populations.

\section{Multidimensional effects in nova outbursts}

The assumption of spherical symmetry in nova models (and in general, in stellar explosions) excludes an entire sequence of events associated with the way in which a TNR initiates (presumably as a point-source ignition) and propagates. The first study of localized TNRs on white dwarfs was carried out by Shara [38] on the basis of semianalytical models. He suggested that heat transport was too inefficient for a flame to spread a localized TNR to the rest of the white dwarf surface (i.e., the diffusively propagated burning wave may require tens of years to extend throughout the whole stellar surface). Therefore, he concluded that localized, volcanic-like TNRs were likely to occur (mainly in $M_{w d} \geq 1.2 M_{\odot}$ white dwarfs). But his analysis, based only on radiative and conductive transport, ignored the major (and crucial) role played by convection on the lateral thermalization of a TNR.

The importance of multidimensional effects for TNRs in thin stellar shells and surface layers (including classical nova outbursts) was revisited by Fryxell \& Woosley [11. They concluded that the most likely situation in nova outbursts involves TNRs propagated by 
small-scale turbulences. From dimensional analysis and flame theory, the authors derived a relation for the velocity of the deflagration front spreading around the stellar surface: $v_{\text {def }} \sim\left(h_{p} v_{\text {conv }} / \tau_{\text {burn }}\right)^{1 / 2}$, where $h_{p}$ is the pressure scale height and $v_{\text {conv }}$ the characteristic convective velocity. Typical values for nova outbursts yield $v_{\text {def }} \sim 10^{4} \mathrm{~cm} \mathrm{~s}^{-1}$ (that is, the flame propagates halfway through the stellar surface in about $\sim 1.3$ days).

The first, pioneering studies that addressed this issue in the framework of multidimensional hydro calculations were performed by Shankar, Arnett \& Fryxell [35, and Shankar \& Arnett [34]. For that purpose, an accreting $1.25 M_{\odot}$ white dwarf was evolved with a 1-D hydro code and mapped into a 2-D domain (a spherical-polar grid of 25x60 km). The explosive event was then followed with a 2-D version of the Eulerian code PROMETHEUS. A 12 isotope network, ranging from $\mathrm{H}$ to ${ }^{17} \mathrm{~F}$, was included to account for the energetics of the explosion. Unfortunately, the subsonic nature of the problem, coupled with the use of an explicit code (with a timestep limited by the Courant-Friedrichs-Levy condition), posed severe limitations on the study, which was restricted to very extreme (rare) cases, characterized by huge $\mathrm{T}$ perturbations of about $\sim 100-600 \%$, in small local regions of the envelope's base. The overall computed time was also extremely small (about 1 second). The calculations revealed that instantaneous, local temperature fluctuations cause Rayleigh-Taylor instabilities. The rapid rise and subsequent expansion (in a dynamical timescale) cools down the hot material and halts the lateral spread of the burning front, suggesting that such local temperature fluctuations do not play a relevant role in the initiation of the TNR (in particular, at early stages). The study, therefore, favors the occurrence of the local volcanic-like TNRs argued by [38].

Later on, Glasner \& Livne [13, and Glasner, Livne \& Truran [14], extended these early attempts. New 2-D simulations were performed with the code $V U L C A N$, an arbitrarily Lagrangian Eulerian (ALE) hydrocode with capability to handle both explicit and implicit steps. As in 3534, a slice of the star $\left(0.1 \pi^{\mathrm{rad}}\right)$, in spherical-polar coordinates with reflecting boundary conditions, was adopted. The resolution near the envelope's base was around $5 \times 5 \mathrm{~km}$. As before, the evolution of an accreting $1 M_{\odot} \mathrm{CO}$ white dwarf was initially followed by means of a 1-D hydro code (to overcome the early, computationally challenging phases of the TNR), and then mapped into a 2-D domain as soon as the temperature at the envelope's base reached $T_{b} \sim 10^{8} \mathrm{~K}$. As in [35 34, the 2-D runs included a 12 isotope network. The simulations revealed a good agreement with the gross picture described by 1-D models (for instance, the critical role played by the $\beta^{+}$-unstable nuclei ${ }^{13} \mathrm{~N},{ }^{14,15} \mathrm{O}$, and ${ }^{17} \mathrm{~F}$, in the ejection stage, and consequently, the presence of large amounts of ${ }^{13} \mathrm{C},{ }^{15} \mathrm{~N}$ and ${ }^{17} \mathrm{O}$ in the ejecta). However, some remarkable differences were also found: first, the TNR was initiated by a myriad of irregular, localized eruptions at the envelope's base caused by convection-driven temperature fluctuations. Hence, combustion proceeds as a chain of many localized flames (not as a thin front), each surviving only a few seconds. Nevertheless, they concluded that turbulent diffusion efficiently dissipates any local burning around the core. As a result, they suggest that the fast stage of the TNR cannot be localized and therefore, the runaway must spread through the entire envelope. Second, contrary to 1-D models, the core-envelope interface is now convectively unstable, providing a source for the envelope's metallicity enhancement through a KelvinHelmholtz instability (a mechanism that bears a clear resemblance to the convective overshooting proposed by Woosley [4]). The efficient dredge-up of CO material from 
the outermost white dwarf layers accounts for a $\sim 30 \%$ metal enrichment in the envelope (the accreted envelope was assumed to be solar-like, without any arbitrary pre-enrichment prescription), in agreement with the inferred metallicites in the nova shells ejected from CO novae. And third, larger convective eddies, extending up to $2 / 3$ th of the envelope's height, with characteristic velocities of $v_{\text {conv }} \sim 10^{7} \mathrm{~cm} \mathrm{~s}^{-1}$, were found in these 2-D simulations. Nevertheless, and despite of these differences, the expansion and progress of the TNR towards the outer envelope was almost spherically symmetric (although the initial burning process was not).

Results from other 2-D simulations were published, shortly after, by Kercek, Hillebrandt \& Truran [24, with the aim to confirm the general features reported in 1314]. In this case, a version of the Eulerian PROMETHEUS code was used. A similar domain (a box of about $1800 \times 1100 \mathrm{~km}$ ) was adopted despite a cartesian, plane-parallel geometry to allow the use of periodic boundary conditions, was chosen. Two resolution runs, one with a coarser $5 \times 5 \mathrm{~km}$ grid (as in [14) and a second with a finer $1 \times 1 \mathrm{~km}$ grid, were performed. Calculations used the same initial model than in Glasner et al. 14] and yield qualitatively similar results but somewhat less violent outbursts (i.e., with longer TNRs and lower $T_{\text {peak }}$ and $\left.v_{\text {ejec }}\right)$, caused by large differences in the convective flow patterns: whereas in [14], a few, large convective eddies dominated the flow, most of the early TNR was now governed by small, very stable eddies (with $l_{\text {max }} \sim 200 \mathrm{~km}$ ) and, accordingly, more limited dredgeup and mixing episodes than in [14] were found. The authors attributed such differences to the different geometry and, more significantly, the boundary conditions adopted in the simulations.

The only 3-D nova simulation to date has also been performed by Kercek, Hillebrandt \& Truran [25]. The run, that adopted a computational domain of 1800x1800x1000 km, with a resolution of $8 \times 8 \times 8 \mathrm{~km}$, revealed flow patterns dramatically different from those found in the 2-D simulations (much more erratic in the 3-D case): mixing by turbulent motions took place on very small scales (not fully resolved with the adopted resolution); peak temperatures achieved were slightly lower than in the 2-D case (a consequence of the slower and more limited dredge-up of core material). Moreover, the envelope attained a maximum velocity that was a factor $\sim 100$ smaller than the escape velocity and, presumably, no mass ejection was expected (except for a possible wind mass-loss phase). In view of these results, the authors concluded that CO mixing must take place prior to the TNR, in contrast with the main results reported by Glasner et al. [14.

\section{To explote or not to explote: discussion and outlook}

Despite the thermonuclear runaway model reproduces the gross observational features of classical nova outbursts, much remains to be done. The following list (by no means exhaustive) outlines different aspects that require further attention:

- Identify the main mechanism (or mechanisms) responsible for mixing at the core/envelope interface.

- Reanalysis of the expected amount of material ejected per event, as compared with the values inferred from observations.

- Better spectra and analysis technics to provide theoreticians with reliable abundance patterns in nova shells. 
- The observation of ${ }^{7} \mathrm{Li}$ in the ejecta accompanying a nova outburst has been extraordinarily challenging. Recently, the presence of this elusive isotope has been claimed for the first time: an observed feature compatible with the doublet at $6708 \AA$ of Li I has been reported in the spectra of V382 Vel (Nova Velorum 1999) 9]. However, its has been argued that such observed feature in V382 Vel may correspond instead to another low-ionization emission centered at around $6705 \AA$ likely the doublet associated with N I [39]. Confirmation of the presence of this isotope in other nova shells would be highly desirable.

- $\gamma$-ray observations of specific gamma-ray signals: that would confirm another of the long-standing predictions of the thermonuclear runaway model, either by detecting line $(478,511$, and $1275 \mathrm{keV})$ or continuum emission.

- More theoretical and experimental work required to shed light into the contribution of novae to the Galactic ${ }^{26} \mathrm{Al}$ content (including studies of nova frequencies and galactic distributions)

- New nuclear physics experiments to reduce the uncertainties associated to key reactions (in particular, ${ }^{25} \mathrm{Al}(\mathrm{p}, \gamma)$ or $\left.{ }^{30} \mathrm{P}(\mathrm{p}, \gamma)\right)$.

- Identification of more nova grain candidates. In particular, it would be interesting to measure sulfur isotopic ratios (never achieved in presolar SiC grains) and to identify as well nova candidates in the oxide grain population.

- More efforts in numerical modeling, specifically in the multidimensional framework. The limited (and somewhat contradictory) efforts to date have to be extended taking advantage of state-of-the-art, massive parallel architectures. First, it is crucial to understand the reason for the differences reported by Glasner et al. [14] and Kercek et al. [24]: critical aspects, such as the specific geometry and the boundary conditions adopted, as well as numerical inaccuracies in the mapping procedure from 1-D models to a multi-D frame can certainly influence the outcome. Moreover, the different flow patterns found when comparing 2- and 3-D simulations suggest that probably only 3-D modeling will bring final answers to our quest for the real nature of nova outbursts.

I would like to thank M. Hernanz, S. Amari, A. Calder, A.E. Champagne, A. Coc, J. D'Auria, J. Dursi, C. Iliadis, S. Shore, S. Starrfield, J. Truran, M. Wiescher, and E. Zinner for many stimulating and enlightening discussions on several topics addressed in this manuscript. This work has been partially supported by the MCYT grants AYA20012360 and AYA2002-0494C03-01, and by the E.U. FEDER funds. Financial support from the catalan AGAUR during a sabbatical leave is also acknowledged.

\section{REFERENCES}

1. A. Alexakis, et al., ApJ 602, 931 (2004).

2. S. Amari, X. Gao, L.R. Nittler, E. Zinner, J. José, M. Hernanz, and R.S. Lewis, ApJ 551, 1065 (2001).

3. S. Bishop, et al., PRL 90, 162501 (2003).

4. H.M.J. Boffin, G. Paulus, M. Arnould, and N. Mowlavi, A\&A 279, 173 (1993).

5. A.G.W. Cameron, ApJ 121, 144 (1955).

6. D.D. Clayton, and F. Hoyle, ApJ 187, L101 (1974). 
7. A. Coc, R. Mochkovitch, Y. Oberto, J.-P. Thibaud, and E. Vangioni-Flam, A\&A 299, 479 (1995).

8. J.M. D'Auria, et al., PR C 69, 065803 (2004).

9. M. Della Valle, L. Pasquini, D. Daou, and R.E. Williams, A\&A 390, 155 (2002).

10. R. Diehl, et al., A\&A 298, 445 (1995).

11. B.A. Fryxell, and S.E. Woosley, ApJ 261, 332 (1982).

12. R.D. Gehrz, J.W. Truran, R.E. Williams, and S. Starrfield, PASP 110, 3 (1998).

13. S.A. Glasner, and E. Livne, ApJ 445, L149 (1995).

14. S.A. Glasner, E. Livne, and J.W. Truran, ApJ 475, 754 (1997).

15. J. Gómez-Gomar, M. Hernanz, J. José, and J. Isern, MNRAS 296, 913 (1998).

16. M. Hernanz, J. José, A. Coc, and J. Isern, ApJ 465, L27 (1996).

17. M. Hernanz, J. José, A. Coc, J. Gómez-Gomar, and J. Isern, ApJ 526, L97 (1999).

18. M. Hernanz, and J. José, this volume.

19. I. Iben Jr., M.-Y. Fujimoto, and J. MacDonald, ApJ 375, L27 (1991).

20. D. Jenkins, et al., PRL 92, 031101 (2004).

21. J. José, and M. Hernanz, ApJ 494, 680 (1998).

22. J. José, A. Coc, and M. Hernanz, ApJ 520, 347 (1999).

23. J. José, M. Hernanz, S. Amari, K. Loddders, and E. Zinner, ApJ, in press (2004).

24. A. Kercek, W. Hillebrandt, and J.W. Truran, A\&A 337, 379 (1998).

25. A. Kercek, W. Hillebrandt, and J.W. Truran, A\&A 345, 831 (1999).

26. A. Kovetz, and D. Prialnik, ApJ, 477, 356 (1997).

27. G.S. Kutter, and W.M. Sparks, ApJ, 340, 985 (1989).

28. M. Livio, and J.W. Truran, ApJ, 318, 316 (1987).

29. J. MacDonald, ApJ, 273, 289 (1983).

30. D. Prialnik, and A. Kovetz, ApJ, 281, 367 (1984).

31. M. Politano, S. Starrfield, J. Truran, A. Weiss, and W. Sparks, ApJ 448, 807 (1995).

32. D. Romano, F. Matteucci, P. Molaro, and P. Bonifacio, A\&A 352, 117 (1999).

33. R. Rosner, A. Alexakis, Y. Young, J. Truran, and W. Hillebrandt, ApJ 562, L177 (2001).

34. A. Shankar, and D. Arnett, ApJ 433, 216 (1994).

35. A. Shankar, D. Arnett, and B.A. Fryxell, ApJ 394, L13 (1992).

36. E. Schatzman, Ann. Ap. 13, 384 (1950).

37. E. Schatzman, Ann. Ap. 14, 294 (1951).

38. M.M. Shara, ApJ 261, 649 (1982).

39. S.N. Shore, et al., ApJ 125, 1507 (2003).

40. S. Starrfield, J.W. Truran, W.M. Sparks, and G.S. Kutter, ApJ 176, 169 (1972).

41. S. Starrfield, J.W. Truran, W.M. Sparks, and M. Arnould, ApJ 222, 600 (1978).

42. S. Starrfield, R.D. Gehrz, and J.W. Truran, in Astrophysical implications of the laboratory study of presolar materials, edited by T. Bernatowicz and E. Zinner, AIP, New York, p. 203 (1997).

43. S. Starrfield, J. Truran, M. Wiescher, and W. Sparks, MNRAS 296, 502 (1998).

44. S.E. Woosley, in Nucleosynthesis and Chemical Evolution, B. Hauck, A. Maeder, and G. Meynet (eds.), Geneva Observatory:Sauverny, p. 1 (1986). 